\title{
La juste mesure
}

\section{Jürg Schlup}

Dr med., président de la FMH

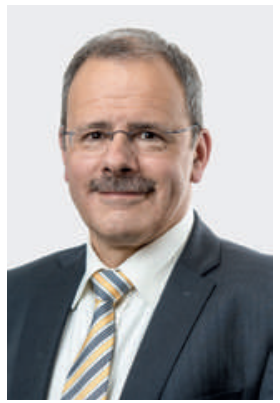

Moins de stress, bouger plus, consacrer plus de temps à sa famille et dépenser moins, sont des résolutions habituelles en début d'année. Bien souvent, elles sont une tentative pour trouver ce qu'on pense être la juste mesure pour la nouvelle année.

Cette juste mesure retient aussi toujours plus l'attention du corps médical lorsqu'il s'agit d'optimiser les prises en charge. Selon une enquête récente [1], 51\% des médecins de premier recours en Suisse estiment que le nombre de prestations fournies aujourd'hui est trop élevé, or ils n'étaient que $38 \%$ de cet avis il y a trois ans. Le volume des prestations s'est-il autant modifié entre 2012 et 2015? Certainement pas, ce résultat montre plutôt la prise de conscience des médecins. Les initiatives du corps médical comme "Smarter Medicine» commencent à porter leurs fruits. Début 2016, le Département de l'intérieur placera la question des prestations injustifiées au centre des débats de la Conférence nationale Santé 2020. Les prestations dites superflues sont prisées par les milieux politiques, qui peuvent annoncer des économies possibles, sans que les citoyens n'aient à en subir de conséquences. Car une prestation superflue ne manquera jamais à personne, ou si peu finalement?

\section{Il s'agit de faire la part des choses entre le «trop" et le "trop peu».}

Malgré les Health Technology Assessments, les guidelines et autres instruments, il n'est pas vraiment facile de déterminer quelles prestations sont inutiles. En dehors de quelques cas évidents, qui ont d'ailleurs été pointés par les sociétés de discipline médicale respectives, nombreux sont les cas pour lesquels une évaluation objective s'avère difficile, voire impossible, parce que les priorités du patient doivent être prises en compte, mais aussi parce que la question se pose d'où situer les limites de l'utilité diagnostique et thérapeutique. C'est malheureusement souvent après que l'on saura, pour le cas particulier, si tel diagnostic supplémentaire ou tel traitement aurait pu être évité.

Avec notre tendance à vouloir exclure le moindre risque, nous nous prêtons à un arrangement plutôt large entre sous-approvisionnement et sur-approvisionnement. Ce sont ces deux notions qu'il s'agit de mettre en relation, comme le révèle l'étude précitée: la proportion de médecins qui estiment que les patients ne bénéficient pas d'un panel suffisant de prestations s'élève en Suisse à $2 \%$ et occupe de loin la queue du classement de tous les pays interrogés. Si les médecins suédois et britanniques sont deux fois moins nombreux à constater une suroffre, 30\% d'entre eux - soit 15 fois plus qu'en Suisse - dénoncent en revanche des problèmes de sous-approvisionnement.

Dans le débat sur l'adéquation des prestations médicales, le corps médical doit faire valoir son expertise: c'est lui seul qui est en mesure de prendre les décisions concernant le traitement.

Trouver la juste mesure est un véritable défi. Il s'agit de faire la part des choses entre le "trop" et le "trop peu». Pour une amélioration judicieuse des soins de santé, il sera essentiel - comme pour les résolutions de fin d'année - de formuler d'abord des objectifs raisonnables et acceptables, et de planifier ensuite comment les atteindre. Par exemple, la campagne «Smarter Medicine» est exemplaire, par sa description des interventions spécifiques à prévoir et la mise en exergue du dialogue entre médecin et patient.

Dans le débat sur l'adéquation des prestations médicales, le corps médical doit faire valoir son expertise: c'est lui seul qui est en mesure de prendre les justes décisions concernant un traitement. Notre travail quotidien consiste à peser les avantages et les inconvénients d'une intervention, en intégrant les objectifs personnels et les préférences de chaque patient.

Toute liberté professionnelle suppose plus de responsabilité. L’assumer signifie savoir porter régulièrement un regard critique sur notre pratique médicale. Dès lors, la tension que suppose la recherche de la juste mesure constitue aussi une condition essentielle pour ressentir la liberté de notre profession et pour rester un partenaire fiable pour nos patients - et ce bien au-delà de l’année 2016.

1 Merçay C. Médecins de premier recours - Situation en Suisse, tendances récentes et comparaison internationale. Analyse de l'International Health Policy Survey 2015 du Commonwealth Fund sur mandat de l'Office fédéral de la santé publique (OFSP). (Obsan Dossier 50). Neuchâtel: Observatoire suisse de la santé; 2015. 